feld-Wolfen und obendrein noch gewählter Vertreter in der KZV sowie LZÄK Sachsen. Auch bei ihm war die aktive Mitgestaltung seines beruflichen Umfelds die treibende Kraft. „Zudem möchte ich die Kolleginnen und Kollegen in unserer Kreisstelle darüber informieren, was genau entschieden wird und auch, warum Dinge eben so entschieden werden, wie sie entschieden werden“, so der Zahnarzt. Der Haken dabei: „Zeitintensiv ist das doch ganz erheblich“, räumt Hofmann ein. Denn für seine Tätigkeiten muss er Zeit für Sitzungen und Besprechungen, aber auch für Organisation, Literaturrecherche und Vorbereitung einplanen - was zum Teil finanziell entschädigt wird. Und so interessant das Ganze auch ist: Hauptberuflich in die Berufspolitik wechseln möchte er nicht.

Das möchte auch Zahnärztin Sara Pourdadasch-Miri nicht. Sie ist Studentenbeauftragte beim Rheinland-Pfälzischen Landesverband (LV) des Freien Verbands Deutscher Zahnärzte (FVDZ). Schon ihr Vater war ein politisch engagierter Mensch, und so war die Politik immer schon ein Bestandteil ihrer Familie. Dazu kommt auch bei ihr der Wunsch, ihren Berufsstand politisch zu unterstützen. Und diese Unterstützung besteht zur Zeit - die frisch aprobierte Zahnärztin ist seit letztem Jahr Mitglied im LV - in der Teilnahme an Sitzungen, und das auch in der stressigen Examenszeit. Was die Zukunft und ein möglicher Wechsel in die Berufspolitik betrift, bleibt sie flexibel: „Zum heutigen Zeitpunkt würde ich nicht wechseln, da mir der Beruf des Zahnarzts sehr viel Freude macht. Aber man soll bekanntlich niemals nie sagen.“

\section{Der Weg in die Berufspolitik}

Was kann man Zahnmedizinern raten, die den Schritt in die Berufspolitik wagen wollen? Zunächst einmal ist wie auch im Beispiel von Frank Hofmann nicht immer ein Berufswechsel nötig, wenn man sich engagieren will. „Gerade junge Kolleginnen und Kollegen mit Interesse für Berufspolitik werden von den (Landes-)Zahnärztekammern mit offenen Armen empfangen. Durch die Akademie für freiberufliche Selbstverwaltung und Praxismanagement wird dafür eine eigene Fortbildung angeboten," weiß Gösling. Wer es intensiver, sprich hauptberuflich will, der sollte sich bei den Kammern und Kassenzahnärztlichen Vereinigungen (KZV) umschauen: „Kammern und KZV sind Körperschaften des öffentlichen Rechts und viele Kollegen wissen gar nicht, welche zahlreiche Möglichkeiten diese Selbstverwaltungen bieten.“, so Gösling. Und auch Beatrice Bade hat einen Rat parat: „Ich empfehle Zahnärzten, die über einen Berufsfeldwechsel nachdenken, sich genau zu überlegen, was sich beruflich verändern soll, wo die eigenen Stärken liegen und ob durch ein berufsbegleitendes Studium fundierte Zusatzqualifikationen im Bereich der Gesundheitswissenschaften erworben werden können."

Swanett Koops

\title{
„Eigenständig aber doch im Team arbeiten - das schätze ich sehr“
}

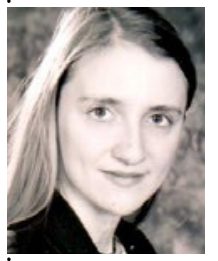

Interview mit Dr. Beatrice Bade, Referentin für die Abteilung Koordination Gemeinsamer Bundesausschuss (G-BA) der Kassenzahnärztlichen Bundesvereinigung (KZBV)

djz: Wann ist bei Ihnen die Idee entstanden, den klassischen Zahnarztberuf zu verlassen und sich einem anderen Berufsfeld zuzuwenden?

Dr. Beatrice Bade: Mit Mitte dreißig kam bei mir das erste Mal der Wunsch nach einer beruflichen Veränderung und neuen Herausforderungen auf. Nach zehn Jahren praktischer Tätigkeit als Zahnärztin kannte ich sowohl die tägliche Arbeit in einer zahnärztlichen Einzelpraxis als auch die von großen Gemeinschaftspraxen. In meiner Arbeit als Zahnärztin habe ich einige Verbesserungspotenziale der zahnmedizinischen Versorgung erkannt. Aus diesem Grund wollte ich jenseits des klassischen Zahnarztberufs nach einer Möglichkeit suchen, mich stärker gesundheitspolitisch zu engagieren.

djz: Welche Voraussetzungen waren für den Wechsel nötig? Brauchten Sie eine zusätzliche Qualifikation?

Bade: Aufgrund meiner langjährigen Berufserfahrung konnte ich umfangreiche Kenntnisse in der vertragszahnärztlichen Versorgung in meine neue Arbeit einbringen. Positiv hat sich sicherlich auch meine Zusatzqualifikation als Fachwirtin im Sozial- und Gesundheitswesen ausgewirkt. Durch meine Pro- motion war ich auch mit Literaturrecherchen und dem wissenschaftlichen Arbeiten vertraut. Des Weiteren sind sicher ein grundsätzliches Interesse an freiberuflicher Selbstverwaltung und Gesundheitspolitik von Vorteil.

djz: Was schätzen Sie an Ihrem neuen/anderen Berufsalltag, was fehlt Ihnen?

Bade: Besonders schätze ich meinen abwechslungsreichen und spannenden Arbeitsalltag in einer sehr angenehmen Arbeitsumgebung. Von besonderem Wert sind für mich die Möglichkeiten, einerseits eigenständig arbeiten zu können und anderseits gemeinsam im Team aktiv an der Gestaltung der vertragszahnärztlichen Versorgung mitwirken zu können. Durch die Festlegung von Gleitzeitmodellen legt die KZBV zudem einen besonderen Wert auf eine ausgeglichene Work-Life-Balance ihrer Mitarbeiter. Hierdurch kann ich meinen Berufsalltag mit meinem Privatleben optimal vereinbaren. Das weiß ich wirklich sehr zu schätzen!

\section{djz: Würden Sie es wieder so machen?}

Bade: Für mich war das Stellenangebot der KZBV eine neue berufliche Chance, die ich ohne großes Zögern ergriffen habe. Und ja - ich würde es jederzeit wieder so machen. Durch meine Tätigkeit als Referentin bei der KZBV habe ich in kürzester Zeit mein Wissen über Selbstverwaltung, Berufs- und Gesundheitspolitik umfassend vertiefen können. Die KZBV ermöglicht mir darüber hinaus die regelmäßige Teilnahme an fachlichen Fort- und Weiterbildungen. 